\title{
A Virtual Reality Environment for Multi-Sensor Data Integration
}

\author{
Scott Papson, Joseph Oagaro, Robi Polikar, John C. Chen, John L. Schmalzel and Shreekanth Mandayam \\ Department of Electrical and Computer Engineering, Rowan University, 201 Mullica Hill Road, Glassboro NJ, 08028
}

\begin{abstract}
Virtual reality (VR) has typically found applications in industrial design, rapid prototyping and advanced scientific visualization. In this paper, we investigate the use of VR for multisensor data integration. We attempt to demonstrate that multiple data types - graphical, functional and measurement can be effectively combined inside of a VR environment. This platform allows the user to rapidly sift through large and complex data sets and isolate features of interest. Furthermore, $V R$ environments can be made to evolve based on system data and user input - this provides the ability to develop scenarios that can be used to make informed decisions. Results demonstrating the effectiveness of this approach are shown using the example of multi-sensor gas transmission pipeline inspection. This work is supported in part by the National Science Foundation award $\# 0216348$.
\end{abstract}

\section{INTRODUCTION}

As modern industrial systems evolve towards complex structures consisting of multiple heterogeneous sensors, there is an urgent need to develop protocols for integrating multisensor data. There have been several attempts at developing comprehensive tools for multi-sensor data fusion, most notably the model developed by the United States Joint Directors of Laboratories (JDL) Data Fusion Group [1]. Artificial neural networks have also emerged as prime candidates for fusing data obtained from multiple sensors [2]. At the same time, virtual reality (VR) platforms have undergone significant evolution, both in terms of ease of use and variety of applications. VR is being routinely used for industrial design, rapid prototyping and advanced scientific visualization. In scientific visualization applications, VR is often times a final process, used solely for the purpose of display [3]. This paper proposes a method by which a virtual reality environment - not only affords superior data visualization, but is also the vehicle for data integration for information obtained from several modalities. When data is integrated in a virtual environment a user then has the ability to immersively navigate, and interact with the data [4]. Additionally, the world can evolve over time to allow the user to interactively evaluate various scenarios.

One specific application, nondestructive evaluation (NDE) of gas transmission pipelines is investigated as a candidate for demonstrating multi-sensor data fusion using VR. There are over 180,000 miles of underground gas transmission pipelines in the United States - these form a critical part of the nation's energy supply infrastructure [5]. A large portion of the pipeline network is over 50 years old and the entire system is subjected to periodic in-line nondestructive evaluation using "pigs" that are conveyed inside of the pipe. The pigs generate magnetic or ultrasonic images indicative of the condition of the pipe-wall; and newer techniques for pipewall interrogation are continually being developed. There also exists significant interest in developing techniques for combining inspection data from multiple NDE methods. The objective of this monitoring is to determine the presence of anomalies such as corrosion, mechanical damage, stresscorrosion-cracking, etc. and assess pipe-wall integrity. Inline inspection generates vast amounts of data - for example, every 100 miles of pipe inspected using magnetic imaging provides $50 \mathrm{~GB}$ of data. When multiple sensor modalities (for example, magnetic, ultrasonic, thermal, acoustic) are used to inspect the same length of pipe, the challenge of data integration becomes formidable. Additionally, information about the pipelines' surrounding environment must be considered after assessing pipe-wall integrity and initiating suitable remediation measures.

\section{OBJECTIVES}

The goal of this research work is to evaluate virtual reality as a candidate for performing multi-sensor data integration. The specific research objectives are:

1. To demonstrate the integration of various data types - graphical, functional, and measurement, inside a virtual environment;

2. To formulate the evolution of the virtual environment based on system data and user input;

3. To demonstrate that the analysis of multi-modal data in a virtual environment is superior to conventional techniques.

As mentioned earlier, the in-line inspection of gas transmission pipelines is used as an application area for evaluating the use of VR in meeting these objectives. Multisensor pipeline inspection data is obtained from the following testing methods - magnetic flux leakage (MFL), ultrasonic testing (UT), thermal imaging, and acoustic emission (AE) testing [6]. This data is input into artificial neural nets to classify data signatures, and predict anomaly geometries. $\mathrm{CAD}$ models of the pipeline are used for registration of the data [7]. The final component of the virtual world is information obtained from the geographic information system (GIS). The GIS information is used to assess environmental 
concerns and provide information about the specific location of the pipeline. The data integration via VR paradigm is illustrated in Figure 1.

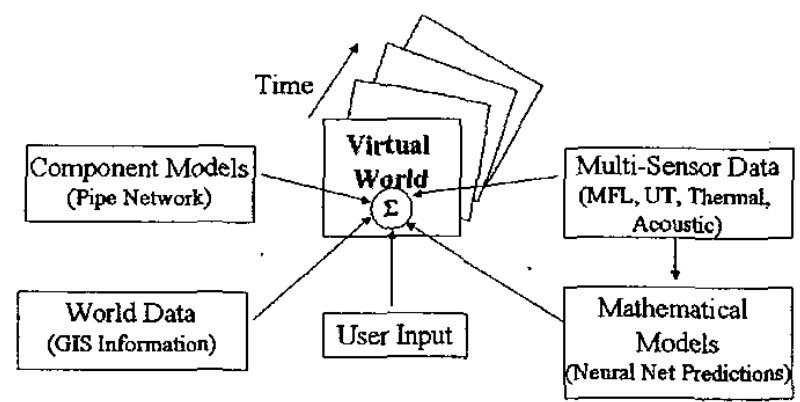

Fig. 1. An overview of the data integration process for the NDE of gas transmission pipelines.

\section{APPROACH}

The various components of the virtual environment that serves as a medium for data integration are described in the following paragraphs.

\section{A. Virtual Environment}

Virtual reality can be defined as any system that allows a user to have immersion, navigation, and interaction. A fully immersive environment is one in which the user is completely engulfed in the virtual world and sensory input for the real world is completely blocked. Navigation in the virtual world allows the user to move to different regions within the world. Finally, interaction is the user's ability to interact with the data. Interaction allows the user to not only see the data, but manipulate it as well [8]. All of these factors contribute to the main thrust of the virtual environment, presence. Presence in a virtual world is the feeling of an accurate depiction of reality in the virtual environment [9].

The creation of presence in a virtual world serves two main purposes. First, it allows the user to visualize data in true three-dimensional representations. Also, it allows the user to visualize phenomena and data such that additional insight is gained. With full control over the virtual world, the designer can create a world such that the user can gain a perspective that would otherwise be difficult if not impossible to obtain [4]. The creation of the virtual world focuses on component representation and component registration. Components with higher detail will create a greater sense of presence. The tradeoff, however, is that increased detail places more demands on the hardware. If the hardware becomes bogged down the world can. lag; the sense of presence will then be lost [10]. Components and data therefore need to be represented with minimum resolution needed for the specific purpose.

\section{B. Data Types}

There are numerous ways data in a virtual world can be described. The most common methods for categorizing data are dividing it according to its representation in the virtual world or its purpose in the virtual world [11]. For most data integration applications the information can be broken down into three categories - graphical, functional, and measurement. Each category can then have several different representations according to the specific software used and desired results.

Graphical objects are traditionally the basis of a virtual world. The graphical objects in the virtual environment represent objects that exist in the actual environment. In inspection applications the representations would include the object being analyzed and any additional objects that are present in the environment. Graphical objects can be modeled using dimensioned drawings, or created visually using a picture or reference object. Graphical objects must be created with detail suitable to fulfill their role for a specific application.

Measurement data is the raw data obtained from any type of sensor. Multi-sensor data integration focuses on representing each data modality individually and in tandem with the other modalities. Depending on the type of data that is obtained, the measurement data can take on a variety of representations [11]. Each data point collected can be represented using a point representation or glyphs. Interpolation between points can be represented through the use of a color slice. A topological representation can be used to vary the data representation spatially as a function of the value. Three-dimensional surfaces can also be created to represent the true form of the data collected.

For each sensor and each modality of data there may not be only one representation that best fits the data. Each application will have specific visualization needs, and the data representations should be tailored to those needs. Multiple representations of a single type of data are also helpful for a user analyzing complex data sets.

Functional components are representations of processed or calculated data. Analyzing raw data is not usually sufficient in most inspection applications. Neural nets or other algorithms can be used to help classify certain areas within the data sets. Components (the graphical objects) can be identified or flagged such that only the areas of interest are analyzed. Evolutionary algorithms can be designed to predict how the current state of the world might evolve over time for a given set of parameters. Analysis using first principles or finite element analysis can also be used to see how measured data compares to theoretical data. Like the measured data, each type of functional data representation will have a representation consistent with the purpose of that data. 


\section{Graphical Data}

Graphical data represents the objects being investigated and the environment in which those objects exist. For this application there are two types of graphical objects being integrated, pipeline components and GIS information. The component models represent the object being investigated and the GIS data conveys information about the environment of the pipeline

There are a number of base graphical objects to represent a pipeline. These include a section of pipe, flange, weld, sleeve, t-section, check valve, ball valve, anchor, and anomalies (crack, pit, etc.). Each component can be modeled using a CAD program. The library of modeled components can then be assembled to create the pipeline that is being inspected. Drawings of the pipeline system can be used to manual piece together the various library components to make an increasingly complex network of pipes. Automated assembly algorithms can also be constructed to allow construction of the pipeline network in the virtual world. The library can also have multiple detail levels for each component. Using lower detail allows for the creation of expansive pipeline networks. When an area of interest is identified, a subsection of the network can be subsequently generated using the components with finer details.

The geographic information system can give a great deal of information about the location of the pipeline network. The GIS can give information about the topography, land usage, location of rivers, roads, buildings, etc. This information can be integrated into the virtual environment such that the user can gain insight into the location details of the pipeline. Under certain conditions remediation measures are determined based on the location of the pipe in question. Integrating GIS information into the virtual environment allows the user to have not only physical location, but additional sociologic information.

\section{Functional Data}

Functional data can be obtained through any type of mathematical model. For this application the functional data is a function of the measured data. Using the data gathered, artificial neural nets can be used to classify the data signatures. The category labels for the output of the neural net are the library components for the graphical library. ANN predictions can be used in two different ways. The predictions can be used to assist in visually classifying the signatures and searching for anomalies within the data collected; also, the predictions can be used to construct a pipeline network. For each neural net prediction the corresponding component piece can be obtained from the graphic library and appended onto the network. This predicted network can be easily compared to the actual network.

If an anomaly is present ANNs can be used to further classify the depth and geometry of the anomaly. Using multiple modalities of data, predictions can be made on the size and shape of an anomaly. This information can be represented in the virtual world, and used to help assess the pipeline integrity.

\section{E. Measurement Data}

Measurement data has been collected using a variety of NDE methods including magnetic flux leakage (MFL), ultrasonic testing (UT), thermal imaging, acoustic émission (AE), and thermal imaging $[12,13]$. A suite of test specimens has been developed to provide a diversity of NDE data when subjected to multi-sensor interrogation. The test specimens were developed to demonstrate the anomalies of pitting corrosion, stress corrosion cracking, and mechanical damage, in the wall of the pipeline.

\section{F. Analysis in Virtual Reality}

One of the benefits of virtual reality is that it allows the user to visualize the data in its natural three dimensional form. Many studies have shown that the immersion, navigation, and interaction in a virtual world allow for a better understanding of complex three dimensional studies $[14,15]$. Studies have also shown that NDE inspection techniques can be performed faster and with more accuracy when using virtual reality [16]. When dealing with large and complex data sets it is therefore imperative to use the techniques that afford the greatest advantage in speed and accuracy.

Using a virtual platform there are infinite ways in which the data can be visualized and subsequently analyzed. Many of the design specifications will vary as a function of the creator and user of the virtual world. The designer has the ability to alter the world in many ways such that analysis of the data is optimized. Factors such as world lighting, color schemes, world dimensions will all affect the analysis and can be optimized for a given application.

For the NDE of gas transmission pipelines it is important to note that the worlds were designed to optimize flexibility to the user. In addition to viewing the pre-generated data representations, the user has the ability to create data representations on the fly. This allows the user to customize any world for a given analysis. Color table properties and transparencies of objects were optimized for specific viewing hardware. Again such parameters may vary from user to user and therefore they too can be varied.

\section{G. Evolutionary Scenarios}

The data obtained from any given inspection of a pipeline inspection is static; it does not vary over time. The condition of the pipeline will however vary over time. Periodic inspection of the pipeline will illustrate this changing condition. It is impractical and expensive to continually monitor and inspect all pipelines. Therefore, evolutionary 
scenarios can be implemented to ease the inspection burden, by predicting the changing conditions of a pipeline. Through user interaction, the virtual world can evolve over time, and illustrate the effects of various scenarios [17].

There are two types of evolution being considered, constrained evolution and non-constrained evolution. In fully-constrained evolution the user would supply a value and a time. Based off of the static state of the world and the user input, the algorithm will predict multiple manners in which this event can take place. Partially-constrained evolution is when the user would specify a value or a time. The world would then evolve towards that particular scenario. Finally, in unconstrained evolution the world uses past and current data to predict the single most likely path for the future. The figure below illustrates unconstrained evolution and fullyconstrained evolution.
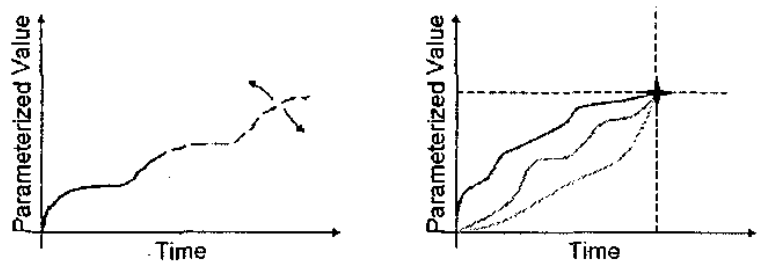

Fig. 2. An illustration of single and multi-path evolution.

Algorithms using four different techniques are being developed to produce the evolution described above. Deterministic algorithms are being investigated to see if the use of first principles affords superior control over an evolutionary path. Auto-regressive moving average (ARMA) models are being used to evolve the world based on past inputs and outputs [18]. Equation 1 below shows the base for an ARMA model.

$u(n)+a_{1} u(n-1)+\ldots+a_{m} u(n-M)=v(n)+b_{1} v(n-1)+\ldots+v_{K}(n-K)$

Where $u(n)$ is the process and $\mathrm{a}$ and $\mathrm{b}$ are the ARMA parameters. Genetic algorithms are being used to base evolution paths on both a fitness score and random mutations within the evolution path itself $[19,20]$. The probability a path will contribute to the next generation is given by:

$$
P(i)=\frac{f_{i}}{\sum_{j=1}^{N} f_{j}}
$$

Where $f_{i}$ is the fitness score for the $\mathrm{i}$-th path, and $\mathrm{N}$ is the total number of paths.

Finally, hidden Markov models (HMMs) are being used to model evolution as a probabilistic sequence of states. Figure 3 illustrates a generic HMM.

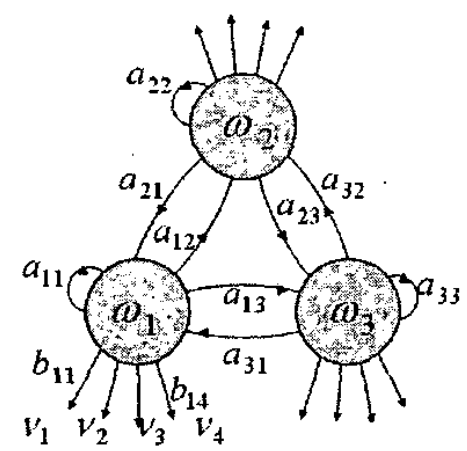

Fig. 3. A HMM model and the transitions between the states.

The figure illustrates three hidden units and the transitions between them. The emission probability of the visible states is also shown, $b_{i j}$ and $v_{j}$ respectively. The following equation can then be described to govern the sequence of states [21].

$$
\begin{aligned}
& a_{i j}=P\left(\omega_{j}(t+1) \mid \omega_{i}(t)\right) \\
& b_{j k}=P\left(v_{k}(t) \mid \omega_{j}(t)\right)
\end{aligned}
$$

\section{RESULTS}

The following worlds were created to implement the procedure as described in the previous section. The Fakespace ImmersaDesk R2, a semi-immersive display system, was the projection system used. The system includes head tracking and a tracked navigation wand. VRCO's vGeo software was used to create the virtual worlds and drive the display system.

\section{A. Graphical}

First, a component library was created. The library included a pipeline anchor, check valve, ball valve, $\mathrm{T}$-section, tap, weld, sleeve, flange, and straight length of pipe. The graphical components were modeled and assembled using SolidWorks. All models were converted to virtual reality modeling language (VRML) files and imported into vGeo. Figure 4 shows three sections of a pipeline network, each having a different set of components. 


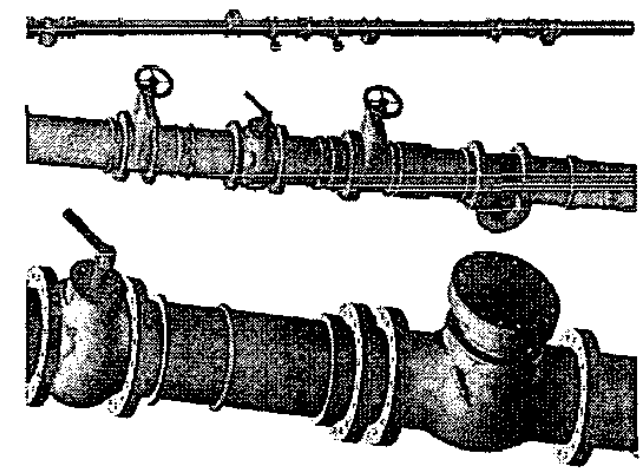

Fig. 4. Pipeline sections that are composed of various library components.

Although this particular assembly was done prior to integration, it is possible to import each piece individually and create the pipeline network interactively.

ESRI's AcrMap was used to obtain certain geographical features. The software generates two-dimensional maps that can be extruded to a third dimension using ESRI's add-on package, 3-D Analyst. Files can then be imported into the virtual world as VRML files. Altemately, two-dimensional pictures can be imported into the virtual reality system. A third dimension, if desired, can then be extrapolated within vGeo. When GIS data is imported as a VRML file, the entire file is one group of data. When imported separately, each portion of data can be turned on and off in the immersive display.

\section{B. Measurement and Functional}

A suite of test specimens was created in order to obtain the NDE signatures from the various testing modalities. The specimens were tested using MFL, UT and thermal imaging. The results of the measurements were then integrated using virtual reality. The figures below show the results obtained using each of the testing methods.

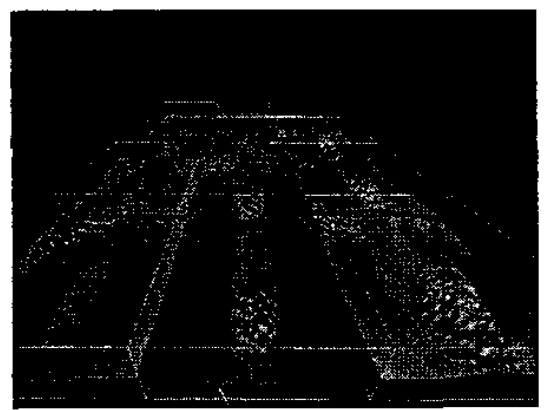

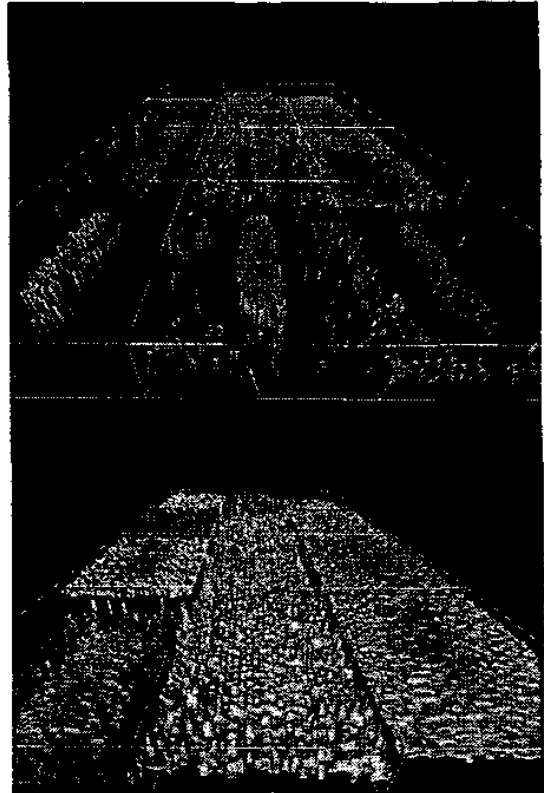

Fig. 5. MFL, UT, and thermal images from the inspection of corrosion pits.

Similarly, the pipe model shown in Fig. 4 was inspected using an MFL pig. Each component piece has a characteristic flux. The flux data was obtained from an MFL pig with 83 sensors around the circumference. The complete set of data for each sensor is represented by one column of data; the measurement of all 83 sensors for a particular distance measurement is represented by each row. This tabular data was then projected onto an irregularly shaped grid. After the circumferential grid was constructed, the grid was distended proportional to the magnitude of the flux observed at each sensor. This is illustrated in Figure 6

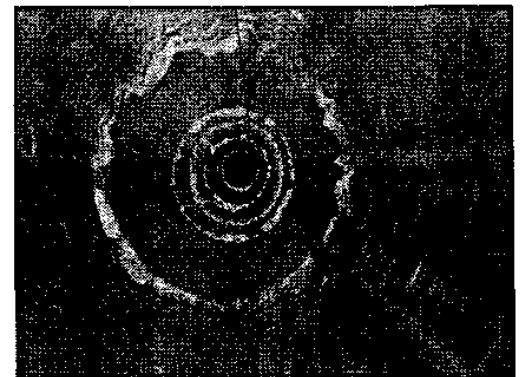

Fig. 6. NUMBER MFL pigging data inside a pipeline.

This information was also fed into a neural network. The ANN classified the flux signatures as one of the component model pieces. The classification results can simply be overlaid onto of the actual flux data. Figure 7 illustrates the integration of the measured data and the predicted classification result. 

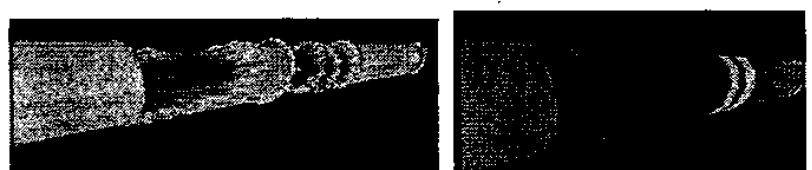

Fig. 7. MFL image obtained from a section of pipe and the corresponding neural net classifications.

This image shows how a simple transparent color slice can be used to easily integrate measured data and functional data. With this simple combination, the results can be quickly and easily verified visually.

\section{Integration}

There are an infinite number of combinations that can be used to represent the various data modalities in a virtual world. In order to view the MFL, UT, and thermal imaging data, color mapped topologies were used. The data from Figure 5 was displayed together to investigate the three data sets at the same time. Figure 8 shows how the aggregate data is integrated.
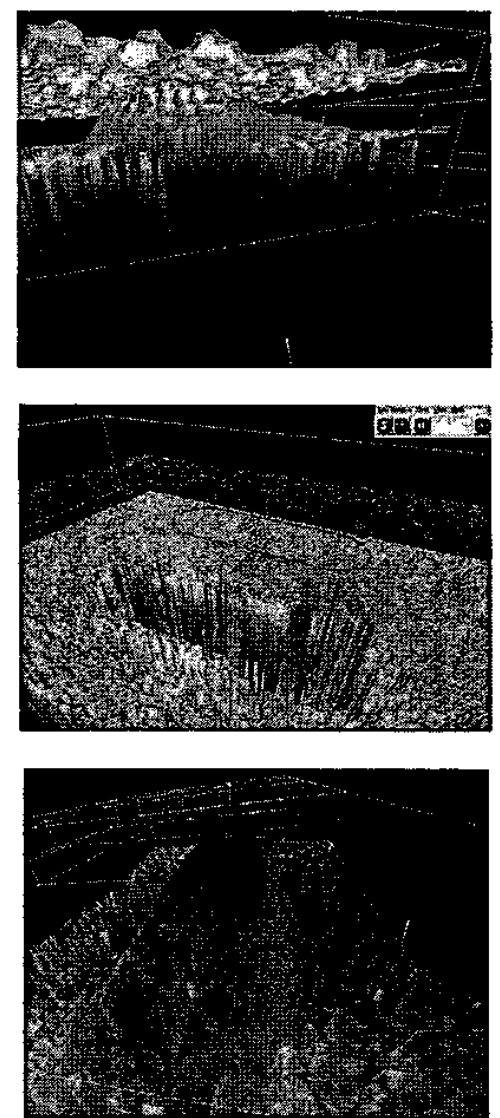

Fig. 8. Various representations of MFL, UT, and thermal data sets integrated in a virtual world.

Contour slices can also be used instead of surface representations to analyze the data. Graphical representations can also be used for reference. Figure 8 shows UT data surface, a thermal contour, and a graphical contour used for reference; also a UT topology, a MFL transparent topology and a thermal contour. The same integration procedures used on the test specimen data was used on the component MFL data. A pipeline network was created and the MFL data was superimposed onto it. Figure 9 illustrates the integration for these two data types.

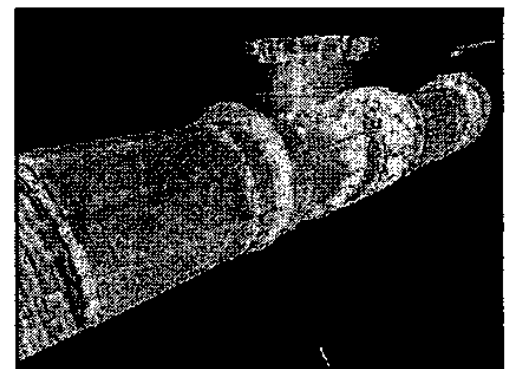

Fig. 9. MFL data registered onto the graphical pipe representation.

Similarly, a pipeline network was created using less detailed components to save graphical memory. The pipeline netwotk was then registered inside of a virtual world. The location information was conveyed through a grid, topography, and simulated GIS data. The integrated world is shown in Figure 10.

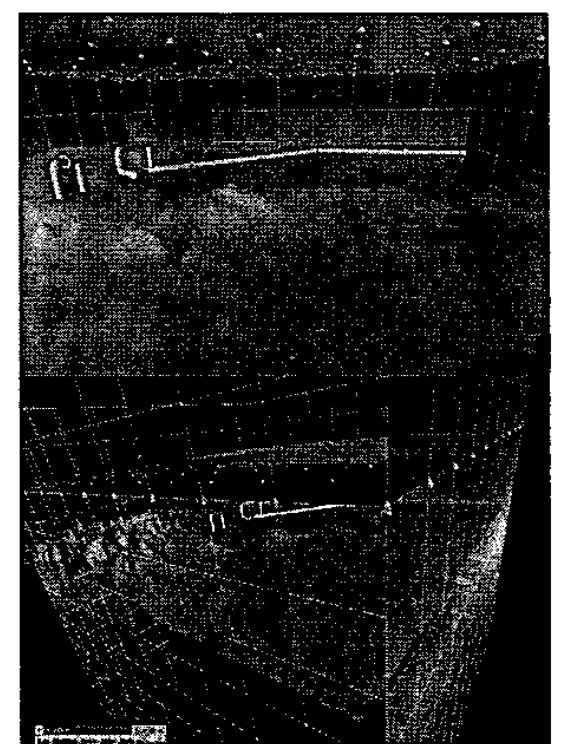

Fig. 10. A view of an integrated environment for a pipeline network.

\section{CONCLUSIONS}

In this paper, we have demonstrated the use of virtual reality as a candidate for multi-sensor data integration. Three types of data, graphical, functional, and measurement, were combined in a virtual environment. Graphical data included pipeline network components, and GIS data. Neural network 
classification results and evolutionary predictions were included in functional data. Finally, multiple modalities of measured data were combined in a virtual world. A virtual environment such as the one described in this paper provides the user with the following powerful capabilities:

1. The user can rapidly sift through large amounts of complex, heterogeneous data sets and isolate events or features of interest in specific geographic or temporal locations;

2. Experiencing the raw (or processed) data and/or predictions in its native 3-D form allows the user to make informed decisions;

3. Virtual environments have the ability to evolve based on resident data or user input - this allows the user to ask "What if?" questions and observe the development of multiple scenarios.

In summary, the VR environment showed significant promise as a method for data integration. The environment showed flexibility and an improved medium for analysis. Future work will focus on improving the integration process by adding more data modalities and making more of the functions automated.

Much of the focus for data integration in virtual reality lies in the ability to alter the state of the world as a function of time, the original state of the world, and user imput. The scenario development portion of this methodology is extremely valuable for a range of applications. Preliminary development has shown one-dimensional evolution in the virtual world. The primary focus is to extend the evolution to an n-dimensional problem. Once an n-dimensional implementation is created, the multiple algorithms will be compared. The evaluation will lead to the development of a single technique that can be used over a range of applications.

The current component assembly is performed prior to data integration. Interactive assembly of library components is also desirable, because of the power it gives to the user. Interactive assembly can also be used in collaboration with ANN classification results to build pipeline networks solely from the data collected. The work in progress is focusing on registration and orientation of the aggregate model. VRML is being used to build the model.

ANN are being used to not only classify the data, but in the presence of an anomaly describe the size and shape of the anomaly. Current research focuses on depth prediction using multiple sources of data. Additionally, efforts are being made to combine heterogeneous data. Imaging data, vector data, and heuristic data all provide some information about the current condition of a pipe wall. The combination of this data should be able to provide an accurate prediction of the condition of the wall.

\section{ACKNOWLEDGMENTS}

This work is supported in part by the National Science Foundation award $\# 0216348$.

\section{REFERENCES}

[1] David L. Hall and James Llinas, Handbook of Multisensor Data Fusion, CRC Press, Boca Raton, 2000

[2] S. Mandayam, L. Udpa, S. S. Udpa and W. Lord, "Signal processing for in-line inspection of gas transmission pipelines," Research in Nondestructive Evaluation, Vol. 8, no. 4, pp. 233-247, 1996.

[3] Andries van Dam, David H Laidlaw, Rosemary Michelle Simpson. Experiments in Immersive Virtual Reality for Scientific Visualization. Computers \& Graphics 2002; 26: 535-555.

[4] T. Hong, A Virtual Environment for Displaying Gas Transmission Pipeline NDE Data, M.S. Thesis, Iowa State University, 1997.

[5] Posakony GJ, and Hill VJ, "Assuring the integrity of natural gas transmission pipelines," Topical Report, Gas Research Institute, November 1992.

[6] Ciocco Michael, An invariance transformation algorithm for defect characterization of ultrasonic signals for the nondestructive evaluation of concrete, M.S. Thesis, Rowan University, 2002.

[7] M. J. Pierce, Scientific visualization of gas transmission pipeline NDE data, M.S. Thesis, lowa State University, 1999.

[8] Linda G. Shapiro, George C. Stockman. Computer Vision. Prentice Hall, 2001.

[9] Mel Slater, Anthony Steed, Yiorgos Chrysanthou. Computer Graphics and Virtual Environment. Addison Wesley, 2002.

[10] Martijn J. Schuemie, Peter Van Der Straaten, Merel Krijn and Charles A.P.G Van Der Mast. Research on Presence in Virtual Reality: A Survey. CyberPsychology \& Behavior 2001; 4(2): $183-$ 201.

[11] Andries van Dam, Andrews Forsberg, David Laidlaw, Joseph La Viola and Rosemary Simpson. Immersive VR for Scientific Visualization: A Progress Report. IEE Computer Graphics and Applications. November / December 2000: 26-52.

[12] Birks Albert S., Nondestructive Testing Handbook: Ultrasonic Testing, American Society for Nondestructive Testing, Inc. Vol. 7 , 1991.

[13] Miller Ronnie, Nondestructive Testing Handbook: Acoustic Emission Testing, American Society for Nondestructive Testing, Inc. Vol. 5, 1987.

[14] Nahm, Sik Lee, Jae Hee Park, Kyung Soo Park. Reality and human performance in a virtual world. International Journat of Industrial Ergonomics 1996; 18: 187-191.

[15] Colin Ware and Glenn Franck. Evaluating Stereo and Motion Cues for Visualizing Information Nets in Three Dimensions. TOG Info Net Vis. April 20, 200: 1-21.

[16] Jeenal Vora, Santosh Nari, Anand K Gramopadhye, Andrew T. Duchowski, Brian J. Melloy, Barbara Kanki. Using virtual reality technology for aircraft visual inspection training: presence and comparison studies. Applied Ergonomics 2003; 33: 559-570.

[17] Trevor Collins. Applying software visualization technology to support the use of evolutionary algorithms. Journal of Visual Languages and Computing 2003; 14: 123-150.

[18] Simon Haykin Adaptive Filter Theory. Fourth Ed. Prentice Hall. 2002.

[19] Darrell Whitley. An overview of evolutionary algorithms: practical issues and common pitfalls. Information and Software Technology 2001; 43: 817-831.

[20] Ibrahim Kushchu, Genetic Programming and Evolutionary Generalization. IEE Transactions on Evolutionary Computation October 2002; 6(5): 431-442.

[21] Richard O. Duda, Peter E. Hart, David G. Stork. Pattern Classification. Second Edition. Wỉley-Interscience. 2001. 